\title{
Numerical simulation of a multi-inlet microfluidic device for biosensing purposes in osteoporosis management
}

\author{
Patricia Khashayar ${ }^{1,2,3} \cdot$ Amir Okhovat ${ }^{4} \cdot$ Hossein Adibi $^{5} \cdot$ Jindrich Windels $^{1} \cdot$ Ghassem Amoabediny $^{3,6}$. \\ Bagher Larijani $^{5}$. Jan Vanfleteren ${ }^{1}$
}

Received: 7 August 2018 / Accepted: 12 June 2019

(C) Springer Nature Switzerland AG 2019

\begin{abstract}
Objectives In this paper, the effect of the position of the inlet and outlet microchannels on the flow profile and the geometry of the recognition chamber for sample pre-treatment in an electrochemical biosensor to be used in osteoporosis management were investigated. Methods All numerical computation presented in this work were performed using COMSOL Multiphysics and Fluent. Simulation was performed for a three-dimensional, incompressible Navier-Stokes flow and so explicit biphasic volume of fluid (VOF) equations were used.

Results In the designed microfluidic system, a pressure-driven laminar flow with no-slip boundary condition was responsible for fluid actuation through microchannels in a reproducible approach. Based on the simulation results, the number of outlets was increased and the angel through which the inlets and outlets were attached to the microchamber was changed so that the dead volume would be eliminated and the fluid flow trajectory, the velocity field and pressure were evenly distributed across the chamber. The Re number in the inlets was equal to 4.41, suggesting a laminar flow at this site.

Conclusion The simulation results along with the fact that the design change was tested using laser ablated tape and a color dye at different steps provided the researchers with the opportunity to study the changes in a fast and accurate but cheap method. The absence of backflow helps with the cross-talk concern in the channels and the lack of bubbles and complete coverage of the chamber helps with a better surface modification and thus better sensing performance.
\end{abstract}

Keywords Simulation $\cdot$ Microfluidic $\cdot$ Biosensor $\cdot$ Microchips

Hossein Adibi and Ghassem Amoabediny contributed equally to this work.

Hossein Adibi

adibi@tums.ac.ir

$\triangle$ Ghassem Amoabediny

amoabediny@ut.ac.ir

1 Center for Microsystems Technology, Imec and Ghent University, Ghent, Zwijnaarde, Belgium

2 Osteoporosis Research Center, Endocrinology and Metabolism Clinical Sciences Institute, Tehran University of Medical Sciences, Tehran, Iran

3 Nanobiotechnalogy Department, Faculty of New Sciences \& Technologies, University of Tehran, Tehran, Iran

4 TeyfPardaz Pishroo, University of Yazd, Tehran, Iran

5 Endocrinology and Metabolism Research Center, Endocrinology and Metabolism Clinical Sciences Institute, Tehran University of Medical Sciences, Tehran, Iran

6 Department of Biotechnology, Faculty of Chemical Engineering, School of Engineering, University of Tehran, Tehran, Iran

\section{Introduction}

The field of microfluidics is characterized by the study and engineered manipulation of fluids at the submillimetre scale [1]. The fluid phenomena at this length scale are different from the macro environment as the relative effect of the force produced by gravity at these dimensions is greatly reduced, and surface tension and capillary forces become more dominant.

Microfluidic technology, thus, is considered as an enabling technology for miniaturized point-of-care (POC) devices, as it allows reduced sample/reagent consumption thanks to accurate and controlled flow process along with parallel processing of samples, reduced reaction time, low production costs allowing for disposability, and portability [2].

In such integrated micro-Total Analysis Systems ( $\mu \mathrm{TAS}$ ), channels perform the essential but simple task of transporting fluids from one place to another such as microchambers for electrode treatment and analyte detection usually as a 
continuous fluid flow. In the development of $\mu$ TAS, it is often necessary to send multiple samples/reagents to the microchambers either to be mixed or to modify the surface consecutively. The flow stream is controlled by adjusting the microchannels' dimensions as well as their position regarding the chamber. Moreover, in order to achieve satisfactory results, it is often needed to achieve uniform and complete filling of the microchamber.

Therefore, the optimum design of the system is important. However, it depends on the final application of the device and the surface modification process. Computer-based simulations are becoming increasingly important in the design of microfluidic devices, as they can predict the behavior of fluid flow in a typical microfluidic system in a short time, yielding results that are virtually identical with that of hours or days of practice [3-6]. In this paper, the effect of the position of the inlet and outlet microchannels on the flow profile and the geometry of the recognition chamber for sample pretreatment in an electrochemical biosensor for osteoporosis management were investigated through a preprocessing step, such as a mathematical simulation.

\section{Experimental procedure}

\section{Micro-chip design}

The pressure-driven microfluidic system was designed to transport the reagents needed for surface modification, the analytes needed to be analyzed, and the probe required for the electrochemical testing to the reaction microchamber. Therefore, flow from the inlets to the micro-channels and then to the microchamber with the help of an additional external force from the syringe pump was necessary. Backflow in other channels was dissuaded as mixture of these solutions outside the chamber might influence the final results negatively. Moreover, uniform and complete filling of the microchamber was needed to improve the performance of the sensor. Moreover, this shows that all the fluids entered the inlet channel leave the system through the outlets without any disturbance.

As medical grade double-sided tape ( $3 \mathrm{M})$ was to be used for the fabrication of the microfluidic platform, the height of the channels was considered equal to the thickness of the tape $(86 \mu \mathrm{m})$. The microchannels had a width of $200 \mu \mathrm{m}$, which was a safe value for serum/blood samples to pass through without clogging or sedimentation.

A $2.9 * 8.9 \mathrm{~mm}^{2}$ microchamber, $86 \mu \mathrm{m}$ deep, equal to the thickness of the tape (volume $=2.2 \mathrm{~mm}^{3}$ ) was designed. It provided the area for the incubation of sample on the electrode surface, and thus the volume was selected to maximize the number of interactions between the agents needed to functionalize the surface as well as target analytes and the functionalized surface.

The diameter of the circular baffles, which were connected to the inlets and outlets, were $1.2 \mathrm{~mm}$. In the implementation of the prototype, Viton O-rings ( $2 \mathrm{~mm}$ inner diameter, $4 \mathrm{~mm}$ outer diameter, $1 \mathrm{~mm}$ thickness) were going to be centered relative to these inlets/outlets to prevent possible leakage. We came up with the design of the intersects of these baffles to the microchannels (diameter of the curve was $1.8 \mathrm{~mm}$ ) based on our in-house expertise; as previous studies in similar designs had shown lower sedimentation and better flow when such intersects were used.

\section{Fabrication method}

The fabrication process of the final device is mentioned elsewhere in an article published by our team [7]. In brief, laser ablation using a focused $\mathrm{CO} 2$ laser beam $\left(9.6 \mu \mathrm{m} \mathrm{CO}_{2}\right.$, mask $1000 \mathrm{um}$ (spot size $100 \mu \mathrm{m}$ ), 1,2 mm/s (7Pulses), $100 \mathrm{~mW}$ ) was applied to pattern the tape and develop the microfluidic manifold. The use of a double-sided tape provided us with a cheap, easy and rapid option to check the efficacy of our simulated design at different steps.

The topmost layer, which served as the cover for the microfluidic channels, was a rectangular piece of COC ( $25.5 \mathrm{~mm}$ by $75.5 \mathrm{~mm}, 1 \mathrm{~mm}$ thickness) mechanically drilled with $1 \mathrm{~mm}$ diameter holes that were used to introduce the sample and buffer solutions as well as to collect the waste. The bottom layer contained the electrodes, and was made of glass.

\section{Numerical simulation}

All numerical computation presented in this work were performed using COMSOL Multiphysics (Finite Element Method) and Fluent (Finite Volume Method). Simulation was performed for a three-dimensional, incompressible Navier-Stokes flow (Newtonian liquids). In this regard, explicit biphasic volume of fluid (VOF) equations was used $\left(\frac{\partial}{\partial t}(\rho \mathrm{v})+\nabla \cdot(\rho \mathrm{vv})=-\nabla \mathrm{p}+\nabla \cdot(\overline{\bar{\tau}})+\rho \mathrm{g}+\mathrm{F}\right.$ a nd $\overline{\bar{\tau}}=\mu$ $\left.\left[\left(\nabla \mathrm{V}+\nabla \mathrm{V}^{\mathrm{T}}\right)-\frac{2}{3} \nabla \cdot \mathrm{vI}\right]\right)$. Regardless of the flow assumptions, a statement of the conservation of mass $\left(\frac{\partial \rho}{\partial t}+\nabla \cdot(\rho \boldsymbol{v})=S_{m}\right)$ is generally necessary. The second order upwind scheme was used to solve discretized momentum equations. Implicit first order differential equations were used to discretized time. Simple scheme was applied to solve the velocity and pressure equations. The accuracy of the calculations was 0.001 .

Three assumptions were made to mimic the actual flow situation: the fluid is Newtonian, no-slip boundary condition along the walls of the microchannel, and the flow within the microfluidic channel is incompressible. Physical values of 


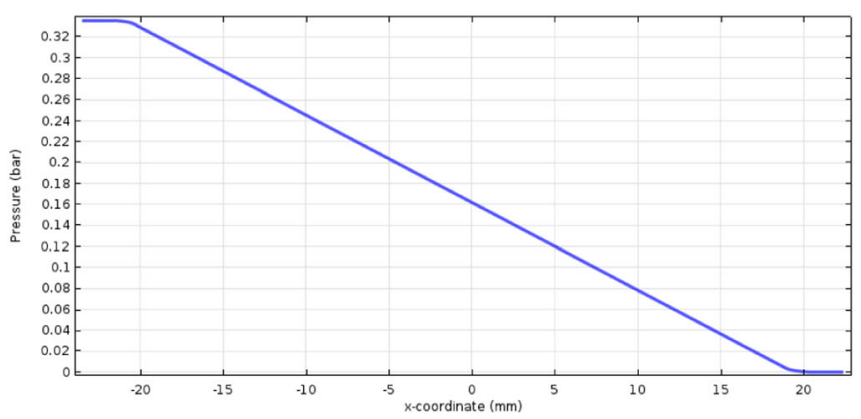

(a)

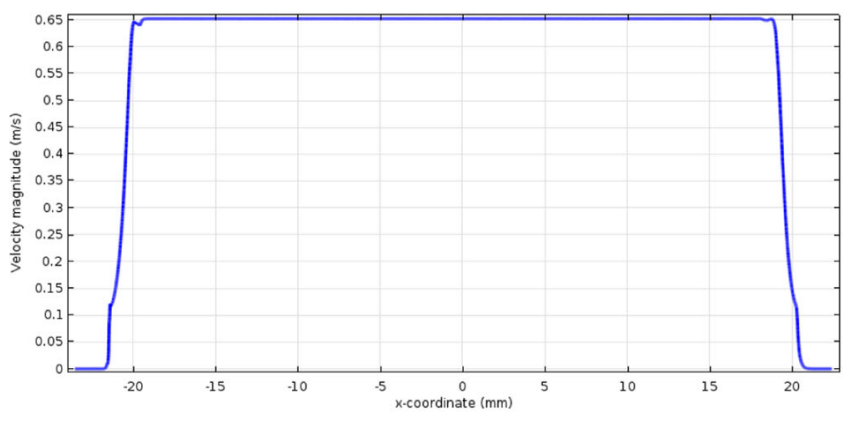

(b)

Fig. 1 a Pressure and $\mathbf{b}$ velocity changes through the microchannel

water (dynamic viscosity: $1.002 \mathrm{e}-3 \mathrm{cP}$; density: $1000 \mathrm{~kg} / \mathrm{m}^{3}$ ) were used in the numerical computation.

The tests were performed in two states:

- Steady state, where the injection time was estimated to be infinite. This profile studies the velocity and pressure at different points of the microchannel. Both outlets were considered open all through the experiments, whereas only one of the inlets was considered open in each set

of tests. As a result, the pressure at the outlet was equal to one atmosphere; and thus the boundary condition at the outlet was considered zero.

- Two phase state, which aimed to study the flow profile in the system. In this simulation, the channels were considered to be filled with air before the first injection. A constant flow rate $(400 \mu \mathrm{L} / \mathrm{min})$ was applied across the crosssection of the inlet and outlet microchannels, pushing the air out of the outlet.

Fig. 2 Velocity contours in the cross-section of the micro channel, showing the velocity along the walls and the center of the microchannel to be zero and maximum, respectively

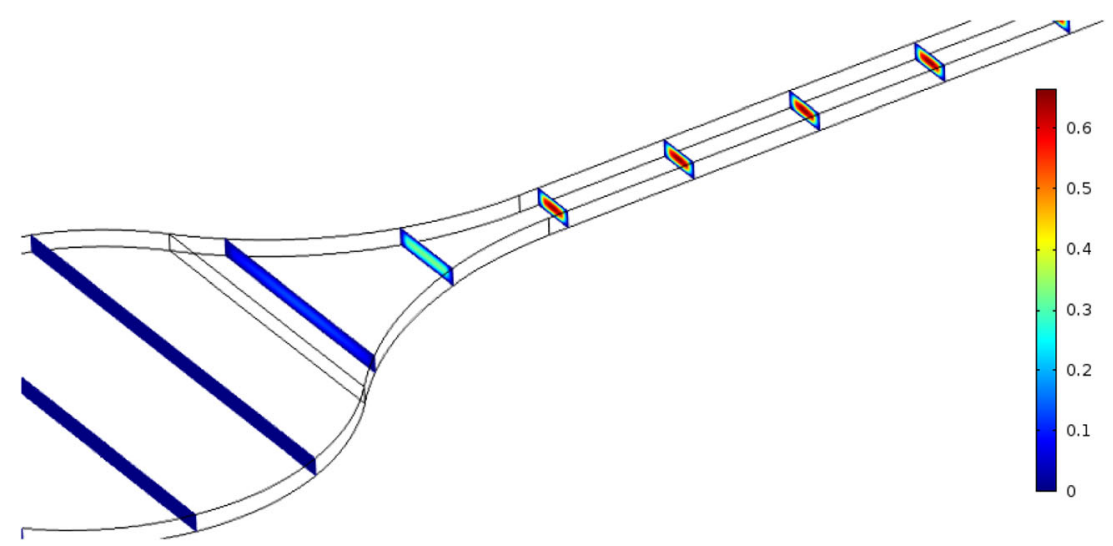

ANSYS

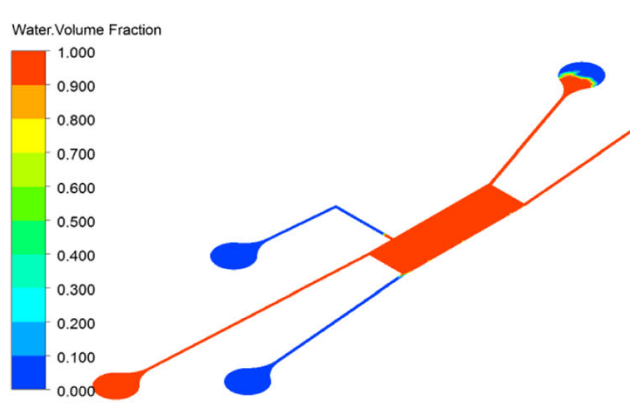

(a)

Fig. 3 Continuous fluid flow trajectories and backflow in other inlets in two designs 


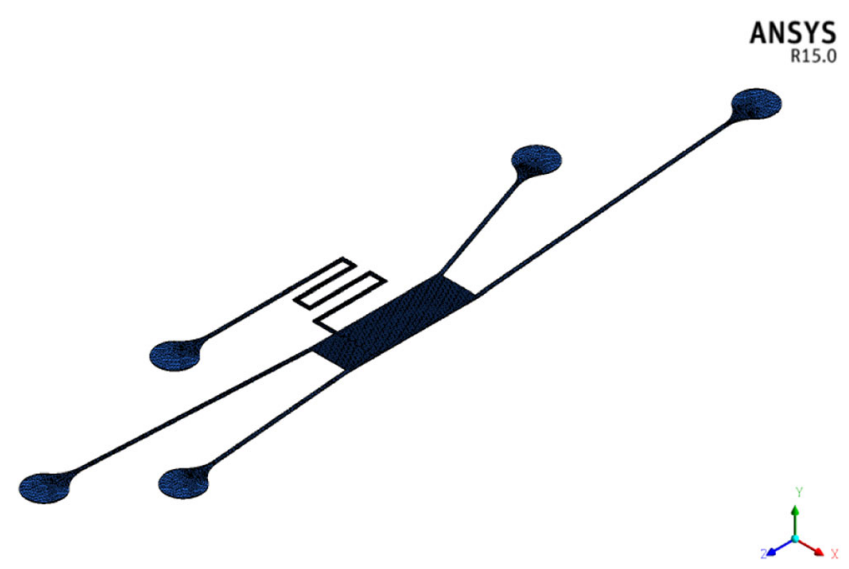

Fig. 4 Geometry modeling in the 2-D cross section of the final optimized design

In order to validate the software setup, a single-channel system ( $40 \mathrm{~mm}$ long, $86 \mu \mathrm{m}$ high and $200 \mu \mathrm{m}$ wide) was used. For this slip length, slip flow is negligible for length scales greater than $1 \mathrm{~mm}$ but must be considered at the microscales [8-10]. It should be noted that as in practice the microfluidic manifold was developed through ablating a double-sided tape, the height of the channels in the simulations was designed to be equal to the thickness of the tape.

The partial differential equations (PDEs) were converted into the algebraic form on a mesh, which defined the geometry and flow domain of the interest. Appropriate boundary and initial conditions were applied to the mesh. Distributions of velocity, pressure and turbulence were determined at different points in space. The pressure drop in the channel is shown in Fig. 1. As it could be seen, the pressure at the beginning and end of the microchannel were constant and the pressure drop through the channel followed a linear drop. This could be explained by the small size of the fully developed length.

The slip velocity at the wall is the most important feature in microscale that differs from conventional internal flow and thus it is very important for designing and optimizing the micro systems. The velocity change through the channel is shown in Fig. 1. In the inlet, the velocity was zero and the pressure was maximum. The considerable velocity increase at the beginning of the channel was due to the mass conversion law and the fact that the fluid entered a small channel with a smaller surface area compared to the inlet. Thereafter the velocity remained constant until it reached the outlet, where a considerable decrease was noted.

When studying the velocity in the microchannel's crosssection, in view of the no-slip condition, the velocity along the walls was zero and the maximum velocity was reported in the center of the microchannel (Fig. 2). The maximum shear stress, on the other hand, was noted on the walls and the minimum in the center of the channel.

The multi-channel network setup was drawn in the Gambit software and studied using the Ansys Fluent. The design was modified in a way that the microchannels and the chamber would be filled completely, the backflow to other inlets was negligible and no dead volume was noted.

\section{Results and discussion}

At microscale level, fluid properties become increasingly controlled and thus when fabricating a microfluidic device, it would be possible to achieve certain specifications simply by changing the design. In microfluidic systems such as ours, pressure-driven laminar flow through positive displacement pumps, such as syringe pumps is responsible for fluid actuation through microchannels in a relative inexpensive and quite reproducible approach. The no-slip boundary condition, referred to a zero fluid velocity at the walls, produces a parabolic velocity profile within the channel, which has significant implications for the distribution of molecules transported within

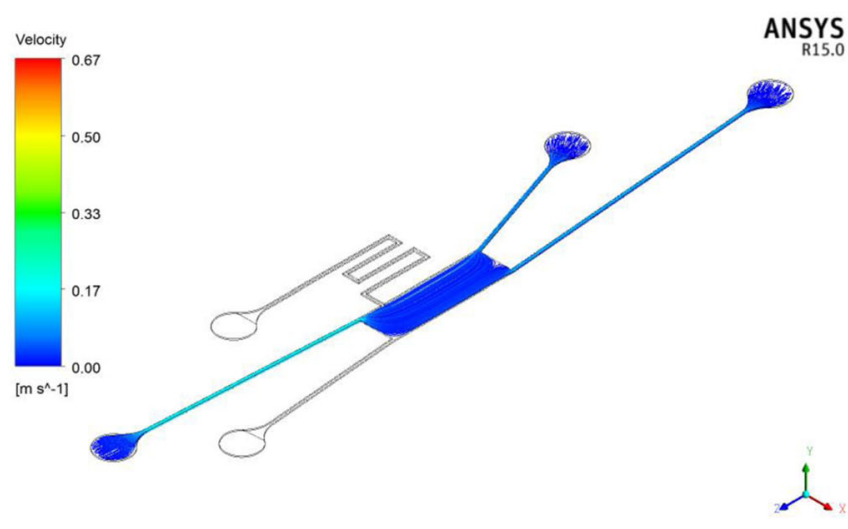

(a)

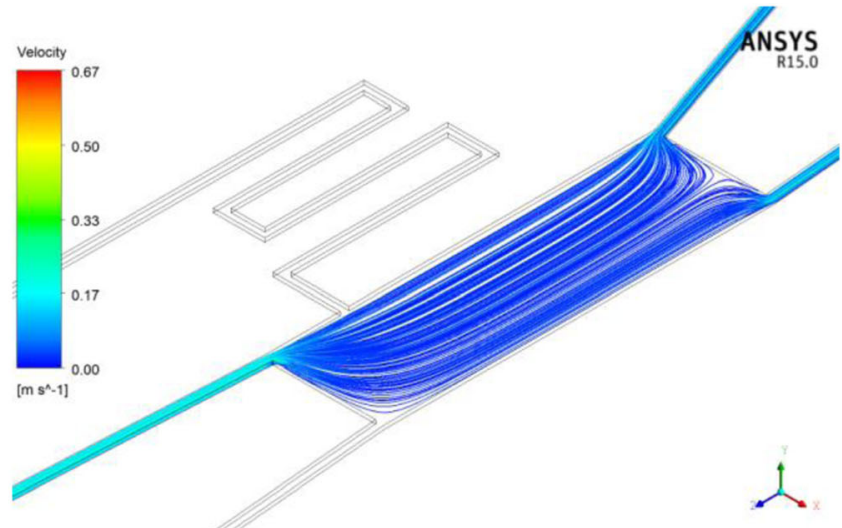

(b)

Fig. 5 Fluid flow in the final design, after adding the extra outlet, showing the microchamber and the microchannels were completely filled out during the injection process 

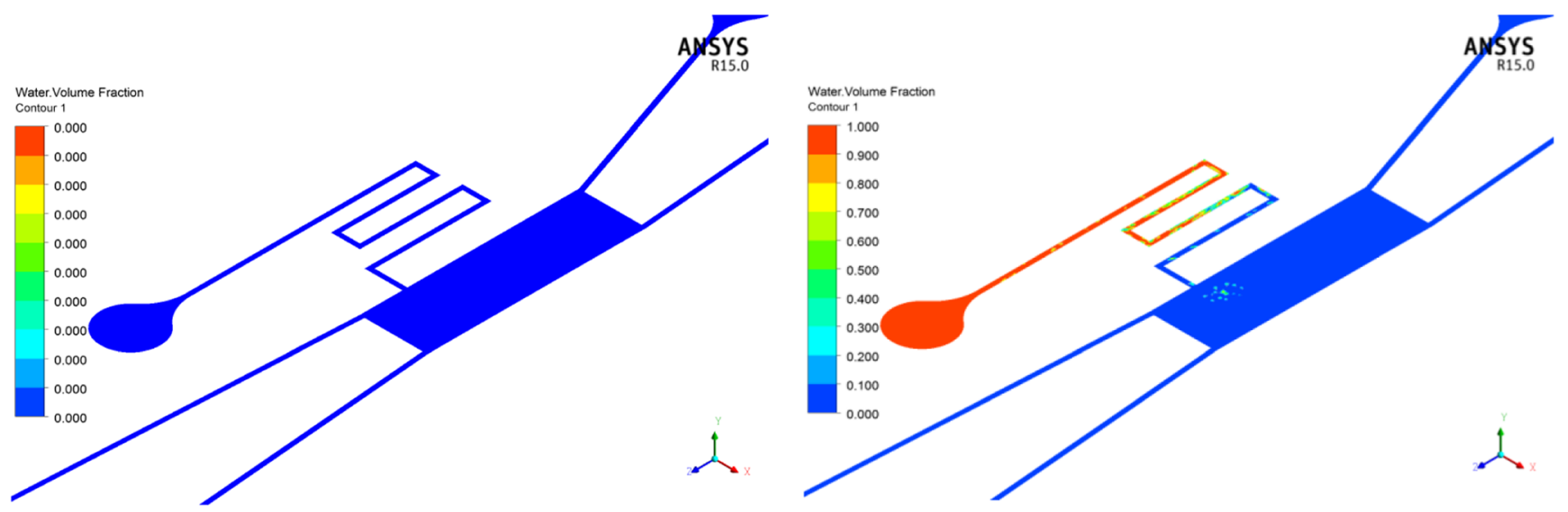

(a)

(b)
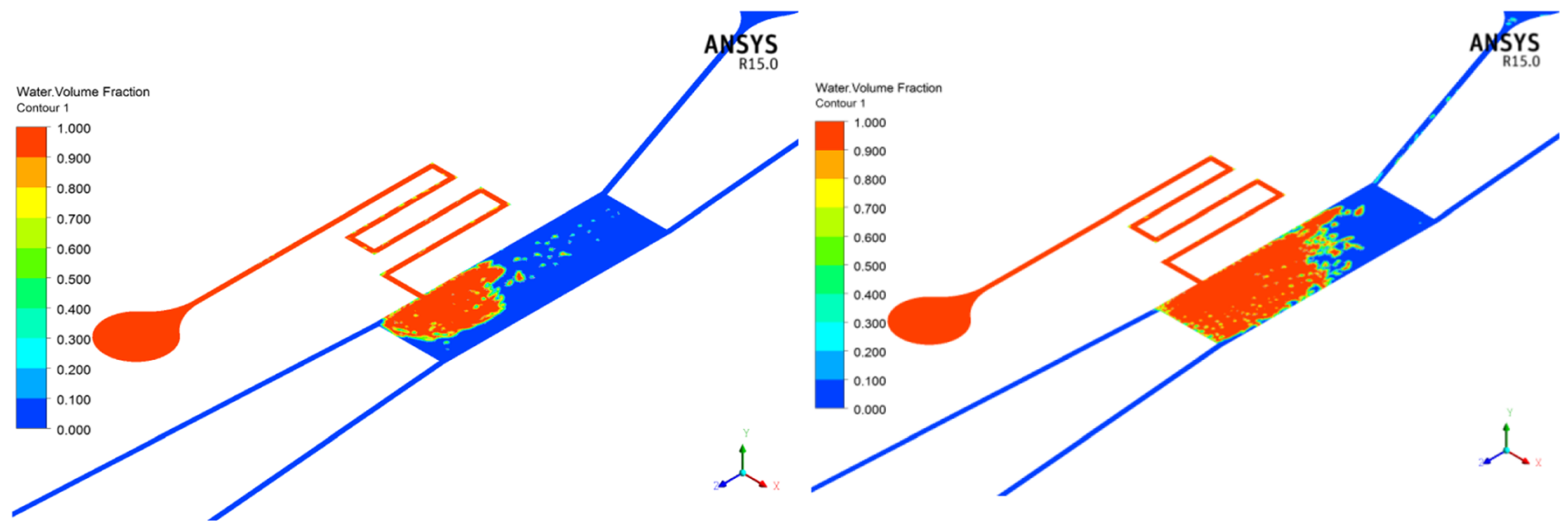

(c)

(d)

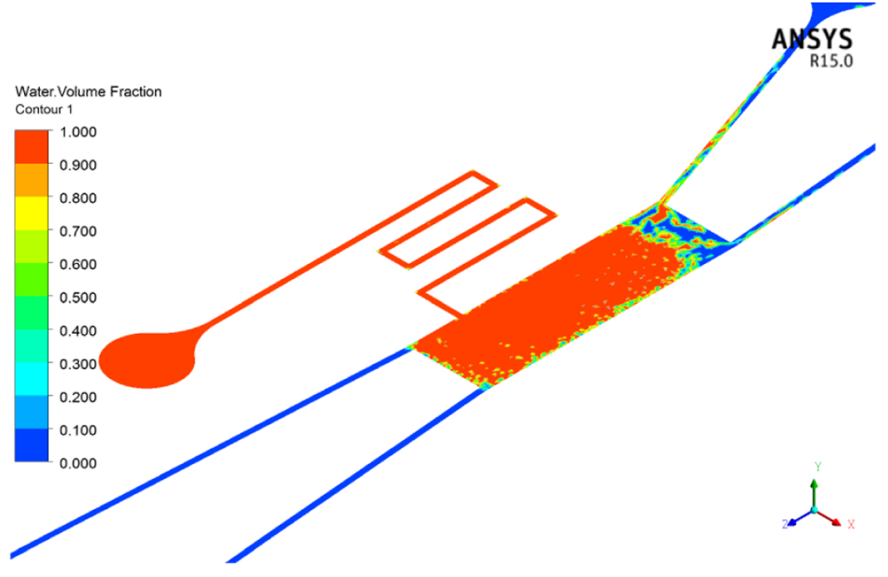

(e)

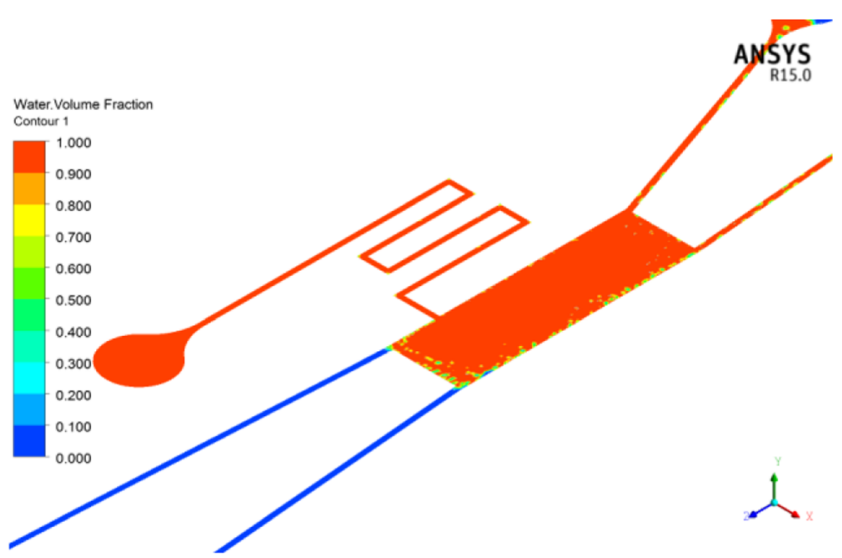

(f)

Fig. 6 Fluid flow trajectories in the final design after injection of the fluid in an inlet showing the filling process at a $0 \mathrm{~s}, \mathbf{b} 0.1 \mathrm{~s}, \mathbf{c ~} 0.2 \mathrm{~s}, \mathbf{d ~} 0.3 \mathrm{~s}, \mathbf{e} 0.4 \mathrm{~s}, \mathbf{f}$ $0.5 \mathrm{~s}, \mathbf{g ~} 0.6 \mathrm{~s}, \mathbf{h} 0.7 \mathrm{~s}, \mathbf{i} 0.8 \mathrm{~s}, \mathbf{j} 0.9 \mathrm{~s}, \mathbf{k} 1 \mathrm{~s}$

a channel. Diffusion controlled how far the fluids could diffuse in the channels [11].

In the first design, the flow entered through three inlets on the left side and exits from a single outlet on the right. The inlets will be used for the injection of the analyte, $\mathrm{Ab}$ complex and detection probe. Diffusion occurred across the microchannels, and there was no convection sideways. It should be noted that in the course of the simulation, dead 

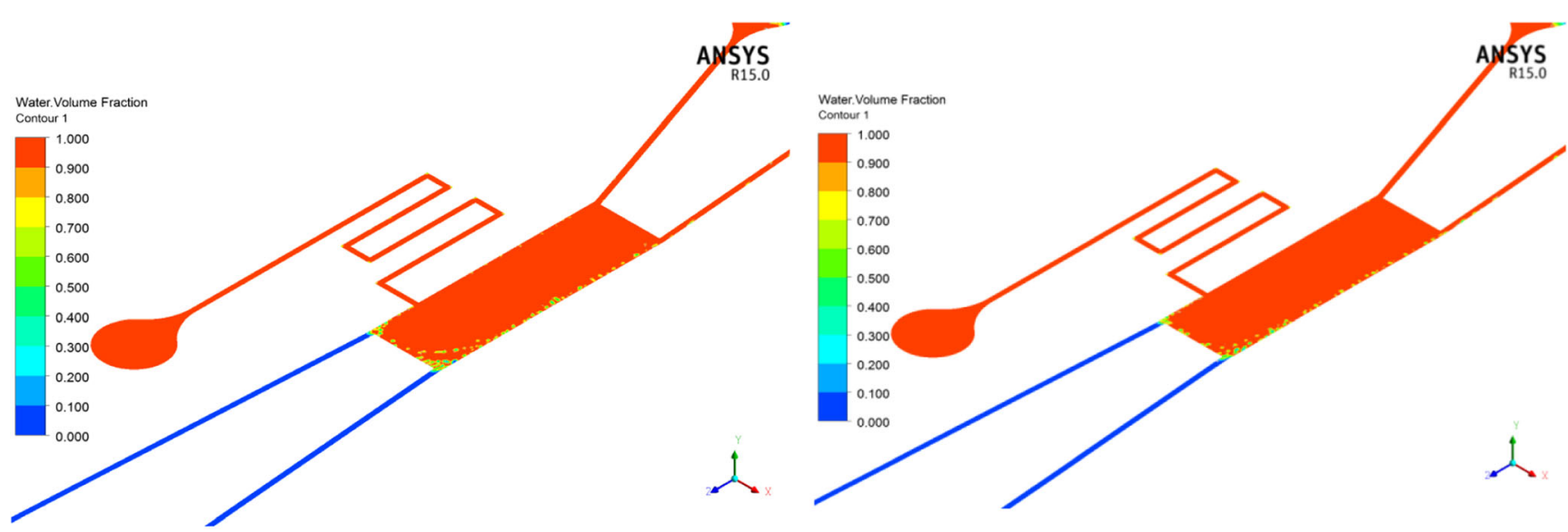

(g)

(h)
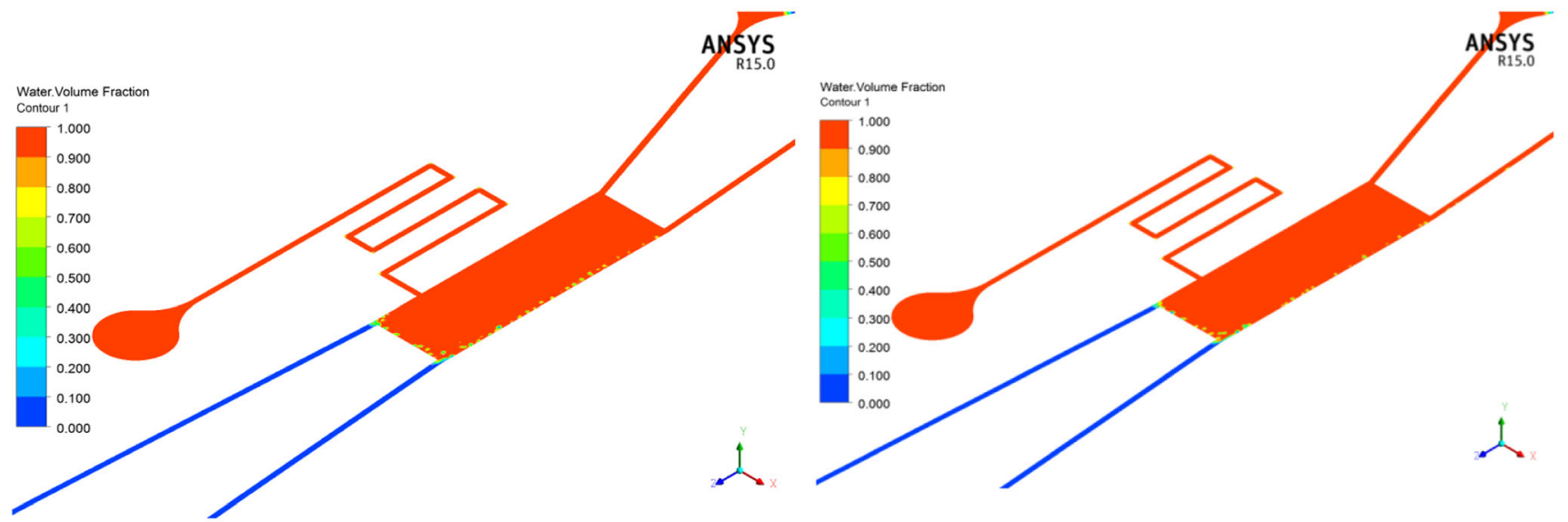

(i)

(j)

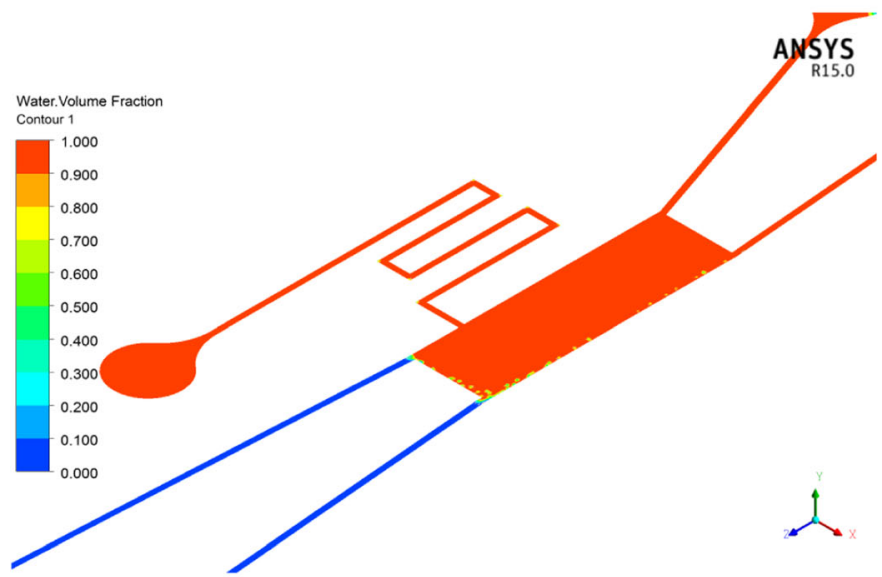

(k)

Fig. 6 continued.

volumes, areas not filled with the fluid, were identified in the square chamber near the inlet and outlet channels, suggesting that the immobilization and washing of the electrodes would not be effective. Based on these observations, a design modification was incorporated, where an additional outlet was added and the angel through which the inlets and outlets were connected to the microchamber was changed so that the dead volume would be eliminated. As mentioned earlier, the design change was tested using laser ablated tape and a color dye at different steps. 
In these tests, backflow of the fluid in other channels, especially the treatment channel was noted. In order to reduce the chance of contamination in the treatment channel, a myriad was added to the treatment channel (Fig. 3). Figure 4 represents the fluid flow trajectories in the final optimized design. In this design, there was no dead volume and the backflow in other inlets was negligible and the fluid flow trajectory, the velocity field and pressure were evenly distributed across the chamber (Fig. 5).

As mentioned earlier, maximum pressure was noted in the inlet. At this point, the gravity force along with the forces due to the syringe pump, were responsible for driving the fluid into the microchannels. A considerable pressure drop was observed when the fluid entered the chamber, where the pressure became equal to that of the closed inlets. As the outlet pressure was zero and the pressure in the chamber was higher than the atmosphere pressure, the fluid was guided through the outlet with a negligible backflow.

The results showed that the chamber was completely filled after the first second, and thereafter the volume fraction of the fluid remained constant. The system was completely filled after a longer injection time.

The microfluidic systems rely on the behavior of fluids in microchannels, and the Reynolds number ( $R e)$ is commonly used to define this behavior [12]. In other words, Re determined the flow instability that induced disturbed and turbulent flow. As the first step, Re was calculated in an inlet:

$$
\begin{gathered}
\mathrm{A}=4.23315 \mathrm{e}-006 \\
\mathrm{p}=0.005890+0.001520=0.00741 \\
D_{h}=\frac{4 A}{p}=\frac{4 * 4.23315 \mathrm{e}-006}{0.00741}=0.00285 \\
\operatorname{Re}=\frac{\rho \mathrm{VD}}{\mu}=\frac{1000 * 0.00157613 * 0.00285}{0.001 * 4.23315 \mathrm{e}-6}=4.41
\end{gathered}
$$

Where, $\rho$ is the fluid density, $\mu$ is the fluid viscosity, $\mathrm{V}$ is the average velocity, $D_{h}$ is the hydraulic diameter, $A$ is the area of the inlet, and $\mathrm{p}$ is the pressure $(\mathrm{Pa})$. The Re number of 4.41 suggests a laminar flow at this site, indicating that the behavior of the micro-scale flow is predictable to a certain extent by

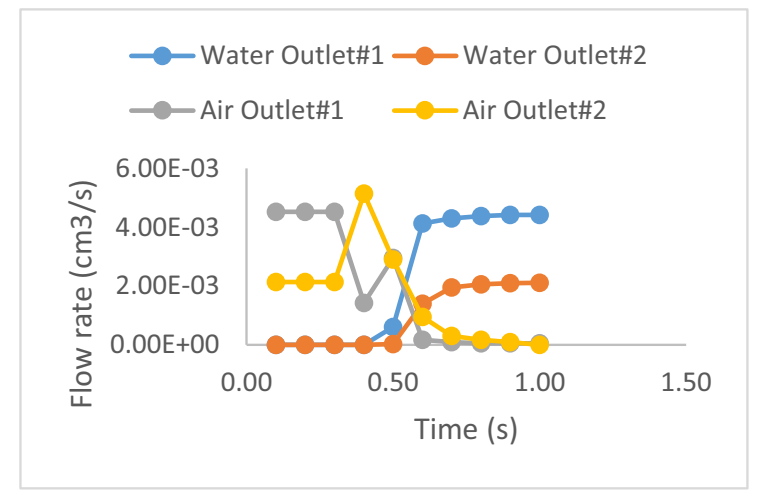

(a) mathematical simulation [13]. A transition from laminar flow to turbulent flow generally occurs when the Re is calculated to be higher than 2000 .

Then, Re was calculated in a cross section of one of the microchannels connecting the inlets to the microchamber. The average output velocity was calculated using Fluent. Maximum Re was expected to be calculated at this point as the highest velocity is also expected here.

$$
\begin{gathered}
\mathrm{A}=1.72657 \mathrm{e}-08 \\
\mathrm{p}=2 *(86 \mathrm{e}-6+200 \mathrm{e}-6)=572 \mathrm{e}-6 \\
\mathrm{D}_{\mathrm{h}}=1.2 \mathrm{e}-4 \\
\operatorname{Re}=\frac{\rho \mathrm{VD} h}{\mu}=\frac{1000 * 0.00157613 * 0.00285}{0.001 * 4.23315 \mathrm{e}-6}=4.41
\end{gathered}
$$

The maximum Re was computed to be 46.3 , confirming a slow and uniform laminar flow in the microchannels. This is while reduced shear stress and vortex flow was observed at the corners of the chamber, which increased the risk of possible sedimentation, pointing out the need for gentle washing after each step.

In order to calculate the pressure needed to achieve an acceptable flow in the microchannels, mathematical simulations were used. As it could be seen in Fig. 6, the chamber was filled within the first second and no change was noted in the fluid until the 6th second, suggesting a constant flow in the outlet.

Figure 7a shows the outlet flow rate for water and air in the first second after injection. It could be seen that the flow rate remained constant after the 0.8 th $\mathrm{s}$.

Figure $7 \mathrm{~b}$ outlines the inlet pressure needed to result in such a flow rate. As it could be seen, the pressure needed for inlets 1 and 2 are the same. This could be due to the similar length of these channels. A higher pressure, however, was needed to allow the fluid to reach the microchamber through the inlet 3; this is mainly because of the shape (bent) and length of the channel.

Along with the mathematical simulations, micro-scale flow visualization plays an important role for understanding the microfluidic flow to develop novel microfluidic devices. As a result, laser ablation was used at several steps to pattern the tape

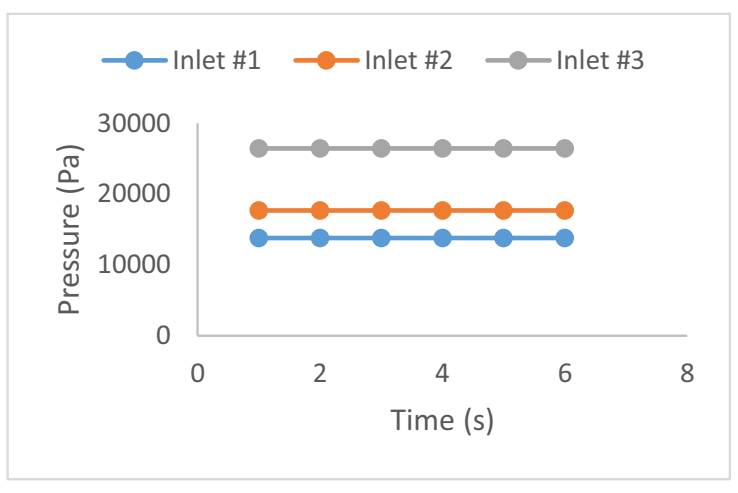

(b)

Fig. 7 a Outlet flow rate for water and air in the first second after injection, b Inlet pressure needed for having a constant flow in the microchannels 
and check the flow using color dye. A cheap disposable microfluidic chip using the final design was then fabricated and tested using human serum to measure concentrations of certain biomarkers necessary for osteoporosis detection. The successful results of these tests are published in an article published elsewhere [14]. This confirms the successful surface modification of the electrodes located in the microchamber thanks to the acceptable flow of the liquids in the chip developed based on this design without any backflow. The comparable results of this chip with that of ECLIA, the state of the art used for measuring these biomarkers, also confirm the desired flow of uncontaminated serum through the channels and complete coverage of the microchamber where the reaction between the serum sample and the electrodes happen.

\section{Conclusions}

We investigated a microfluidic model describing the flow of liquid in microchannels reaching microchamber for surface treatment of the electrodes located in the chamber and analyte measurement. The model is composed of three inlets and two outlets positioned along the microchamber to reduce the risk of backflow and result in uniform and complete filling of the system.

Using such simulation techniques and testing the design using laser ablated tape and a color dye, the final model is easily adaptable and modifiable based on the final application of the microfluidic device. The final design proposed in this study provides researchers with the opportunity to modify the surface of the electrodes located in the recognition chamber for the biosensing purposes simply and accurately using small amounts of reagents. The absence of backflow helps with the cross-talk concern in the channels and the lack of bubbles and complete coverage of the chamber helps with a better surface modification and thus better sensing performance. Similar techniques could be used in future studies for microfluidic chips designed for various applications with more complicated integrated systems.

Acknowledgments This project was supported by the grant no. 92030410 at Iran National Science Foundation (INSF).

\section{Compliance with ethical standards}

Conflict of interest The authors declared that they have no conflict of interest.

\section{References}

1. Sackmann EK, Fulton AL, Beebe DJ. The present and future role of microfluidics in biomedical research. Nature [Internet]. Nature Research; 2014 Mar 12 [cited 2017 Feb 7];507(7491):181-189. Available from: http://www.nature.com/doifinder/10.1038/ nature13118. Accessed Dec 2018
2. Duncombe TA, Tentori AM, Herr AE. Microfluidics: reframing biological enquiry. Nat Rev Mol Cell Biol [Internet]. Nature Research; 2015 Aug 21 [cited 2017 Feb 7];16(9):554-67. Available from: http://www.nature.com/doifinder/10.1038/ nrm4041. Accessed Dec 2018

3. Wang J, Rodgers VGJ, Brisk P, Grover WH. Instantaneous simulation of fluids and particles in complex microfluidic devices. Cao BY, editor. PLoS One [Internet]. Public Library of Science; 2017 Dec 21 [cited 2018 Dec 31];12(12):e0189429. Available from: https://dx.plos.org/10.1371/journal.pone.0189429

4. Low WS, Kadri NA, Abas WAB bin W. Computational fluid dynamics modelling of microfluidic channel for dielectrophoretic BioMEMS application. ScientificWorldJournal [Internet]. Hindawi Limited; 2014 [cited 2018 Dec 31];2014:961301. Available from: http://www.ncbi.nlm.nih.gov/pubmed/25136701

5. Zhao Z, Fisher A, Cheng D. Numerical Simulation in Microfluidics and the Introduction of the Related Software. Microfluidics: Fundamental, Devices and Applications [Internet]. Weinheim, Germany: Wiley-VCH Verlag GmbH \& Co. KGaA; 2018 [cited 2018 Dec 31]. p. 147-74. Available from: https://doi.org/10.1002/ 9783527800643.ch4

6. Hashim U, Diyana A, Adam T, Perlis I, Kayangan M. Numerical simulation of microfluidic devices.

7. Khashayar P, Amoabediny G, Larijani B, Hosseini M, Van Put $\mathrm{S}$, Verplancke R, et al. Rapid prototyping of microfluidic chips using laser-cut double-sided tape for electrochemical biosensors rapid prototyping of microfluidic chips using laser-cut double-sided tape for electrochemical biosensors. J Braz Soc Mech Sci Eng. 2016.

8. Barrat J-L, Bocquet L. Large Slip Effect at a Nonwetting FluidSolid Interface. Phys Rev Lett [Internet]. American Physical Society; 1999 Jun 7 [cited 2018 Dec 31];82(23):4671-4. Available from: https://doi.org/10.1103/PhysRevLett.82.4671

9. Ngoma GD, Erchiqui F. Heat flux and slip effects on liquid flow in a microchannel. Int J Therm Sci [Internet] 2007 Nov [cited 2018 Dec 31];46(11):1076-1083. Available from: http://inkinghub. elsevier.com/retrieve/pii/S1290072907000555. Accessed Dec 2018

10. Yang J, Kwok DY. Effect of liquid slip in electrokinetic parallelplate microchannel flow. J Colloid Interface Sci [Internet]. 2003 Apr 1 [cited 2018 Dec 31];260(1):225-233. Available from: http://www.ncbi.nlm.nih.gov/pubmed/12742054. Accessed Dec 2018

11. Soleymani A, Kolehmainen E, Turunen I. Numerical and experimental investigations of liquid mixing in T-type micromixers. Chem Eng J. 2008;135:S219-28.

12. Stone HA, Kim S. Microfluidics: Basic issues, applications, and challenges. AIChE J [Internet]. Wiley Subscription Services, Inc., A Wiley Company; 2001 Jun [cited 2017 Feb 9];47(6):1250-4. Available from: https://doi.org/10.1002/aic.690470602

13. Jeon W, Shin CB. Design and simulation of passive mixing in microfluidic systems with geometric variations. Chem Eng J. 2009;152(2):575-82.

14. Khashayar P, Amoabediny G, Larijani B, Hosseini M, Verplancke $\mathrm{R}$, Schaubroeck D, et al. A multiplexed microfluidic platform for bone marker measurement: a proof-of-concept. Micromachines. $2017 ; 8$.

Publisher's note Springer Nature remains neutral with regard to jurisdictional claims in published maps and institutional affiliations. 